\title{
SEMG based Intention Identification of Complex Hand Motion using Nonlinear Time Series Analysis
}

\author{
Yaxu Xue \\ School of Electrical and Mechanical Engineering \\ Pingdingshan University \\ Henan, China \\ Email: yaxu.xue@whut.edu.cn
}

\author{
Zhaojie Ju \\ School of Computing \\ University of Portsmouth \\ Portsmouth, UK \\ Email: zhaojie.ju@port.ac.uk
}

\author{
Kui Xiang \\ and Jing Chen \\ School of Automation \\ Wuhan University of Technology \\ Wuhan, China
}

\begin{abstract}
This paper proposes a hand motion recognition system for classifying different complex hand motions based on Surface Electromyography (SEMG). By defining ten common hand motions, the SEMG signals are recorded based on a SEMG capture device. A series of signal processing methods, including signal denoising, and feature extraction are analyzed to acquire the SEMG features. A trained Random Forest (RF) algorithm is used for the classification of ten different hand motions. The experimental results show that the proposed hand motion recognition system has a higher classification accuracy for identifying different hand motions.

Keywords-Hand motion; SEMG; Nonlinear time series; Random forest
\end{abstract}

\section{INTRODUCTION}

Over the years, Surface Electromyography (SEMG) as myoelectric signal, has been widely applied for assistive and rehabilitation technology, human robot collaboration, teleportation,etc [1]-[3]. It is generated by the muscle cells which are electrically or neurologically activated, and detected by using surface electrodes [4]. The SEMG has become a hotspot worthy of research, and is being used for prosthetic hand control, arms, medical abnormalities detection, neuromuscular problems diagnosis and so on.

Generally speaking, interference and cross-talk introduce non-linearity into the biomedical signals. Some existing techniques such as domain feature and frequency domain used for SEMG feature extraction are based on the linear characteristic of the signal [5]. This approach may be compromised by interference and cross-talk from the adjacent muscles. Moreover, the SEMG signal is extremely influenced by different subjects, recording environment. Increasing efforts have been made in analysing the complexity and non-linear nature of SEMG signals. Ju et al. [6] proposed non-linear feature extraction and classification method to evaluate different hand motions, and concluded that recurrence plot (RP) and Fuzzy Gaussian Mixture Models (FGMMs) as nonlinear analysis methods are suitable for dynamical characteristics representation of SEMG signal. Lu et al. [7] used hidden Markov model (HMM) and Expectation Maximization (EM) algorithms to recognize ten hand manipulation signals. Xiaochuan Yin and Qijun Chen [8] presented a nonlinear time alignment method with deep autoencoder to extract spatio-temporal features for human action recognition. In a more recent study Ding et al. [9] proposed a parallel multiple-scale convolution architecture for gesture recognition by using convolution neural networks. The results show that with in some reasonable range of features, human hand motions could be recognized with a satisfactory recognition rate from SEMG signal, but the approach suffers from some issues such as training of data and classification errors.

Due to the highly nonlinear and non-stationary nature of the SEMG signal, it is necessary to use a nonlinear dynamic method to analyze with SEMG signal feature. Lyapunov Exponent (LE) method has been used widely with SEMG signals. The LE based methods mainly include Local Wolf Lyapunov Exponent (WLE) [10], Rosenstein Lyapunov Exponent (RLE) [11], Kantz Lyapunov Exponent (KLE) [12], Local Lyapunov Exponent (LLE) [13] and Maximal Lyapunov Exponent (MLE) [14]. WLE, RLE and KLE have certain antiinterference ability, whereas LLE is easily affected by initial conditions and noise. By contrast, MLE has the ability to estimate the amount of chaos in a system, and demonstrate the changes of the dynamical variables of complex hand motion [15]. In summary, the MLE method is more suitable for the intention analysis of complex human hand motion based on SEMG signals.

This paper proposes a nonlinear time series method for analysing SEMG signal. Different chapters of this paper are organized as follows: firstly, the hand motion recognition system is designed, and the methods of implementation, including data collection, signal denoising, feature extraction and random forest classifier are also presented in detail in Section II; then, Section III presents the detailed experimental analysis and discussion; finally, the conclusion of this paper, as well as the further direction are concluded.

\section{Methods}

\section{A. Hand Motion Recognition System}

The SEMG based complex hand motion system were proposed to design and develop a robust classification scheme for prosthetic hand control. In order to classify different complex hand motions efficiently, the complete schematics diagram of the proposed SEMG signal classification framework consists of five main parts: human hand motions, SEMG device system, 
signal processing, trained RF classification and hand motion recognition, as shown in Fig.1.

The implementation process of the gesture recognition system is: firstly, ten complex human in-hand motions are designed; secondly, the SEMG device system is used for SEMG signal acquisition when the subject performs different tasks; then, the signal processing method is applied to extract features from SEMG signal; finally, RF algorithm is adopted to train a classification model based on the selected SEMG features, and evaluate the performance of the proposed complex hand motion system.



Fig. 1: Hand motion recognition framework

\section{B. Dada Collection}

According to the different operation tasks, the appropriate number of contact points and fingers are determined by the pose of the object, and the hand grasp strategies such as transfer, translation and rotation are combined to achieve stable manipulation. For better recognizing hand motions, this paper reports a new thought that has taken ten representative hand motions as recognizing motion set. Fig.2 shows the demonstration of each complex motion.

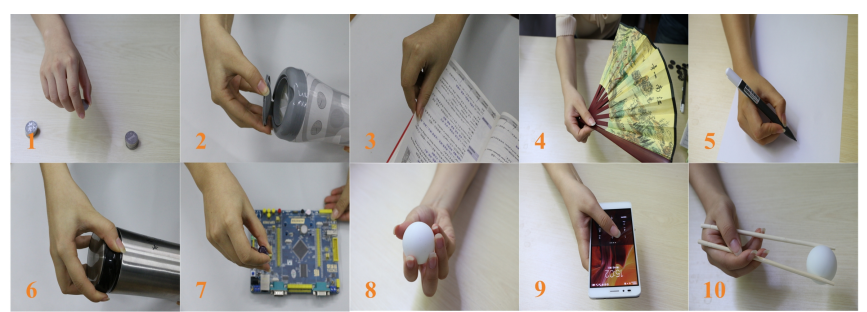

Fig. 2: Ten defined complex hand motions
Ten healthy subjects, eight males and two females, aged 25 \pm 3.6 , height $1.68 \pm 0.13 \mathrm{~m}$ and weight $63 \pm 15.4 \mathrm{~kg}$ were chosen to perform these motions. All subjects signed the informed consent forms, and the local research ethics committee proved the experiments. Before doing the experiments, the subjects were trained to perform the required tasks. The right forearm position of subject was uniformly required to remain on the experiment table. Each subject would perform ten times per each motion. After completing a set of motions, subject would have a rest for two minutes to avoid muscle fatigue.

An SEMG capture device consisting a master appliance, an electrode sleeve, a bluetooth adapter and some connecting wires, was used to collect the SEMG signal. The SEMG signals were acquired from 5 forearm muscles ( flexor carpi radialis, flexor carpi ulnaris, flexor pollicis longus, flexor digitorum profundus, and extensor digitorum). Six channels were selected to record the original signals. The signal resolution is 16 bits and the signal sample is $1024 \mathrm{~Hz}$.

\section{Signal Denoising}

Due to external environmental disturbances and the influence of data acquisition device, SEMG signals are affected by various noises during acquisition and transmission. Therefore, it is necessary to denoise the original SEMG signal for feature extraction. Empirical Mode Decomposition (EMD) algorithm was firstly proposed by Dr. Huang and used for data processing and data mining [16]. This algorithm is a new method for analyzing nonlinear and non-stationary data, which has more advantages than Wavelet Analysis (WA). It decomposes complicated time series data into a finite set of oscillatory modes called Intrinsic Mode Functions (IMFs), and obtains instantaneous frequency data.

Assuming that each channel SEMG signal is $S_{i}(t),(i=1, \cdots, 6)$, and is processed by EMD. Then, a series of averaged IMFs $c_{j}$ is derived. Eq. (1) is calculated as:

$$
S_{i}(t)=\sum_{j=1}^{n} c_{j}+\delta_{n}
$$

Where $n$ numbers of IMFs $c$ are extracted, $\delta_{n}$ is the residue of data $S_{i}(t)$.

\section{Feature Extraction}

The Lyapunov exponent of a chaotic system is a average rate of infinitesimally close trajectories in phase space. In general, the $\mathrm{N}$-dimensional system produces an exponential spectrum, which has $\mathrm{N}$ numbers of Lyapunov characteristic indices. The largest value among all indices is called MLE. It is not only an important indicator for distinguishing chaotic attractors, but also a quantitative description of initial value sensitivity. The definition of MLE is as follows:

$$
\lambda_{\max }=\frac{1}{t} \sum_{k=1}^{M} \operatorname{In} \frac{L\left(t_{k}\right)}{L\left(t_{k-1}\right)}
$$


Where $L\left(t_{k}\right)$ represents the distance of the nearest zeros at $t_{k}, M$ is the total steps, and $t$ is a sampling time.

The distance of the adjacent orbits has typically multiplied by forecasting error on a log scale to acquire the MLEs of the whole IMFs. Its definition is as follows:

$$
p(k)=\frac{1}{N t} \sum_{n=1}^{N} 1 b \frac{\Gamma_{n}(k)}{\Delta_{n}}
$$

Where $N$ is the amount of phase points, $\Delta_{n}$ is the distance between phase point $X_{n+1}$ and $X_{n}\left(X_{n+1}\right.$ is the nearest point from $\left.X_{n}\right), \Gamma_{n}(k)$ is the distance between $X_{n+1}$ and $X_{n}$ which is after $k$ evolutionary step length. In the $p(k)-k$ graph line, the slope of straight line is MLE. In our experiments, the length of overlapping windows is $500 \mathrm{~ms}$ and the increment between windows is $125 \mathrm{~ms}$. The MLE features were obtained for the RF classifier by using Eqs. (1) and (2).

\section{E. Randon Forest}

As a supervised machine learning algorithm, the random forest algorithm was first proposed by Ho to build a collection of decision trees with the combination of Breiman's "Bootstrap aggregating" idea [17], [18]. It has been widely used for classification and regression tasks in some fields, such as biomedical, e-commerce and stock markets. The following RF model is used to acquire the instruction sets, as shown in Fig.3.

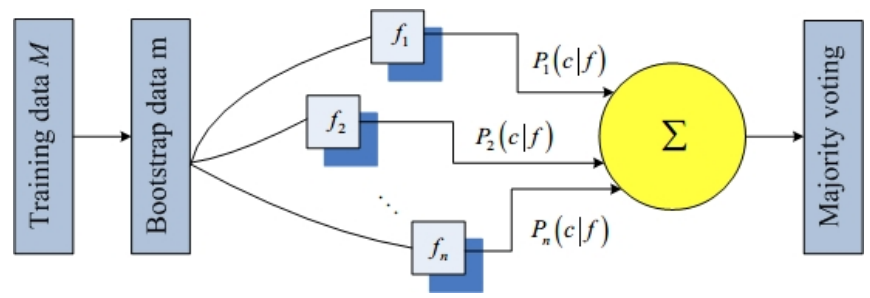

Fig. 3: ML algorithm Principle

A random forest is a classifier consisting of a collection of tree-structured classifiers $\left\{h\left(x, v_{k}\right), k=1,2, \cdots\right\}$, where the $\left\{v_{k}\right\}$ are independent identically distributed random vectors and each tree casts a unit vote for the most popular class at input $x$. The basic principle of RF is: firstly, m numbers of subdata sets are obtained from training data $\mathrm{M}$ based on sampling with replacement; then, m sub-models are built using sub-data sets, and optimal segmentation is constructed with randomly selected features; finally, the results of sub-models are voted and integrated to get the final result.

\section{RESULTS ANALYSIS}

\section{A. Parameters Setting And Analysis}

The delay time (DT) and the embedding dimension (ED) are the two key parameters for LE calculation. Hence, in our experiments, the DT value $\tau=5$ is selected for the phase space reconstruction, the $\mathrm{ED}$ value $\varepsilon=8$ is used for the topologically reconstruction. TABLE I shows the MLEs of SEMG signals which were computed by using the above mentioned methods in Section II. It can be seen that the ten hand motions have different mean value (MV) and standard deviation (SD). For the same motion, the values of MV and SD are significantly different. Lower MLEs values are critical to improve the classification accuracy of the RF classifier. Note that the results of channel 2 are smaller and smoother compared to other channels. Overall, the obtained MLEs values of SEMG signals are suitable for MLE features extraction, which in turn provided appropriate training sample.

\section{B. Recognition With Random Forest}

In order to evaluate the performance of hand motion recognition system, Fig.4 shows the confusion matrix for the ten hand motions using trained RF classifier. The average recognition rate of each motion, and the classification errors between any two motions are exhibited. As can be seen from the confusion matrix, the recognition rates of all hand motions are over $90 \%$, indicating good classification accuracy of the system. Motions 8 and 10 have the lowest recognition rate of $90 \%$, while the recognition rates of motion 4 and 5 are up to $94 \%$. It is worth noting that the error rates of motion 2 and 6 , motion 6 and 8 have reached $6 \%$. In addition, the error rate of motion 8 and 4 also reached $5 \%$. The total recognition rate is $91.67 \%$, but the high error rate shows that the hand motion recognition system might be able to realize higher classification accuracy by using more SEMG features.

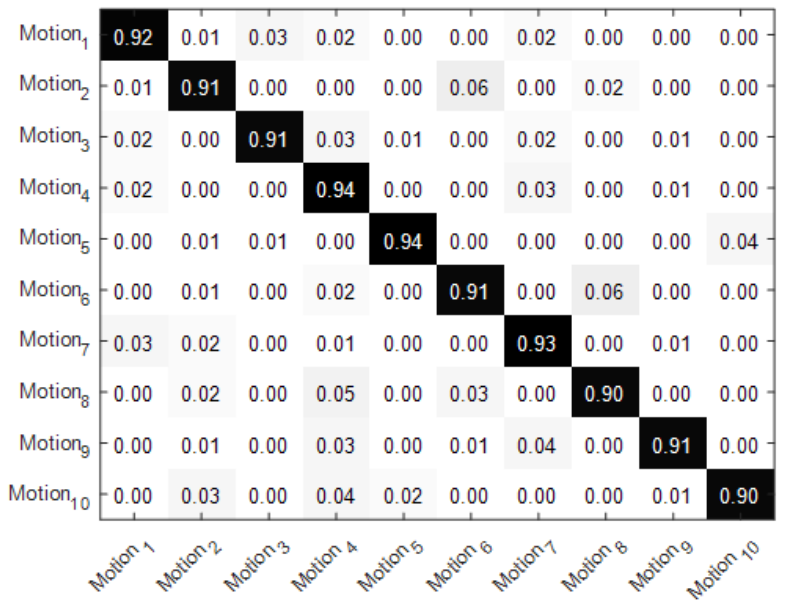

Fig. 4: Confusion matrix for the ten motions using ML

Considering the differences in human operating habits and grasping force, for the same motion, different subjects can use different manipulation methods for stable grasp, further more the SEMG signals are more susceptible to the subjects. Hence, it is necessary to study the effects of individual variation of different subjects on the recognition results of different motions. Fig.5 shows the recognition rates of all motions with different subjects, and exhibits significant difference. Although the unified requirement of manipulation methods of the ten defined hand motions are performed when extract the SEMG signals, the classification results of hand motions are obviously influenced by individual variation. As can be clearly seen from 
TABLE I

THE MLEs OF SEMG SIHNALS

\begin{tabular}{lllllll}
\hline Hand motions & $\begin{array}{l}\text { Channel 1 } \\
\text { MV } \pm \text { SD }\end{array}$ & $\begin{array}{l}\text { Channel 2 } \\
\text { MV } \pm \text { SD }\end{array}$ & $\begin{array}{l}\text { Channel 3 } \\
\text { MV } \pm \text { SD }\end{array}$ & $\begin{array}{l}\text { Channel 4 } \\
\text { MV } \pm \text { SD }\end{array}$ & $\begin{array}{l}\text { Channel 5 } \\
\text { MV } \pm \text { SD }\end{array}$ & $\begin{array}{l}\text { Channel 6 } \\
\text { MV } \pm \text { SD }\end{array}$ \\
\hline motion 1 & $0.1159 \pm 0.0147$ & $0.0982 \pm 0.0125$ & $0.1754 \pm 0.0227$ & $0.1636 \pm 0.0214$ & $0.0867 \pm 0.0243$ & $0.1584 \pm 0.0135$ \\
\hline motion 2 & $0.1233 \pm 0.0176$ & $0.1018 \pm 0.0137$ & $0.2091 \pm 0.0198$ & $0.1519 \pm 0.0187$ & $0.1153 \pm 0.0139$ & $0.1942 \pm 0.0196$ \\
\hline motion 3 & $0.1139 \pm 0.0124$ & $0.1233 \pm 0.0261$ & $0.1649 \pm 0.0114$ & $0.1723 \pm 0.0172$ & $0.1055 \pm 0.0126$ & $0.1841 \pm 0.0113$ \\
\hline motion 4 & $0.1457 \pm 0.0211$ & $0.1256 \pm 0.0179$ & $0.1883 \pm 0.0201$ & $0.1449 \pm 0.0157$ & $0.1069 \pm 0.011$ & $0.204 \pm 0.0229$ \\
\hline motion 5 & $0.1253 \pm 0.0249$ & $0.1141 \pm 0.0118$ & $0.2037 \pm 0.0195$ & $0.1452 \pm 0.0119$ & $0.0935 \pm 0.0117$ & $0.1995 \pm 0.0264$ \\
\hline motion 6 & $0.1146 \pm 0.0133$ & $0.1327 \pm 0.0154$ & $0.1871 \pm 0.0154$ & $0.1693 \pm 0.012$ & $0.101 \pm 0.0174$ & $0.2271 \pm 0.0177$ \\
\hline motion 7 & $0.1662 \pm 0.0211$ & $0.1097 \pm 0.0113$ & $0.1993 \pm 0.0226$ & $0.1834 \pm 0.0258$ & $0.1295 \pm 0.0187$ & $0.1743 \pm 0.0134$ \\
\hline motion 8 & $0.1571 \pm 0.0185$ & $0.1389 \pm 0.0175$ & $0.1769 \pm 0.0189$ & $0.1694 \pm 0.0162$ & $0.1227 \pm 0.019$ & $0.2359 \pm 0.0341$ \\
\hline motion 9 & $0.182 \pm 0.0171$ & $0.1176 \pm 0.0191$ & $0.1735 \pm 0.0177$ & $0.1694 \pm 0.0162$ & $0.1227 \pm 0.019$ & $0.1984 \pm 0.0165$ \\
\hline motion 10 & $0.1368 \pm 0.0169$ & $0.1154 \pm 0.0142$ & $0.2113 \pm 0.0243$ & $0.1876 \pm 0.0218$ & $0.1264 \pm 0.114$ & $0.1938 \pm 0.0221$ \\
\hline
\end{tabular}

Fig.5, all recognition rates are maintained at around $90 \%$. Subjects 6 has the highest recognition rate of $94.23 \%$ for all motions, while the lowest recognition rate for subject 5 , which is $89.12 \%$. In addition, for subject 2 and 8 , the fluctuating range of motion classification is higher, and the maximum error rates are $12.67 \%$ and $20.45 \%$, respectively.

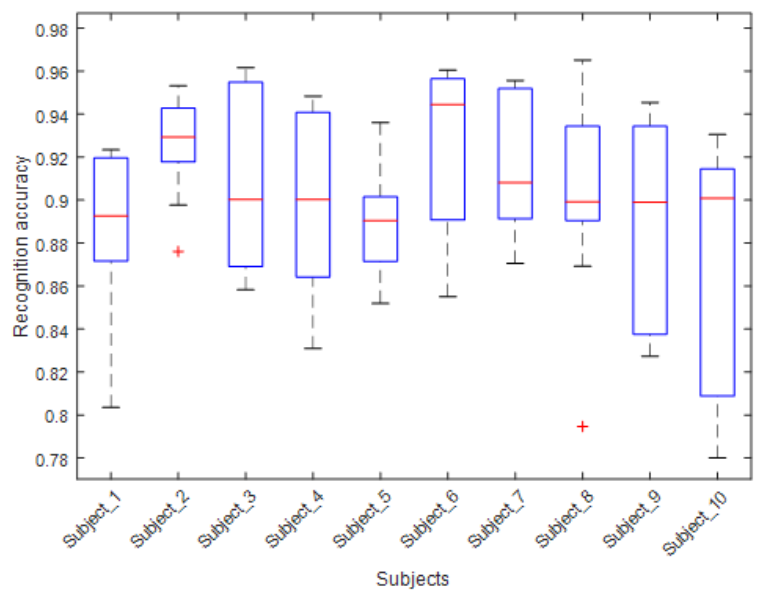

Fig. 5: Recognition rates with different subjects

\section{Comparison of Classical Approaches}

To evaluate the classification performance of the RF classifier, it is necessary to compare it with other machine learning algorithms. Support Vector Machine (SVM) as a generalized linear classifier, divides the samples to obtain the maximal interval by finding a hyperplane, thus realizing the classification of data samples [19]. Neural Network (NN) is a kind of machine learning technology that realizes artificial intelligence by simulating human brain, and has been widely used for information processing and pattern recognition [20]. Two domain features, namely, mean absolute value (MAV) and root mean square (RMS), were selected as features samples. The definitions of the two features are:

$$
\begin{aligned}
M A V & =\frac{1}{N} \sum_{i=1}^{N}\left|x_{i}\right| \\
R M S & =\sqrt{\frac{1}{N} \sum_{i}^{N} x_{i}^{2}}
\end{aligned}
$$

The parameters taken to create and optimize the classifier models are shown in TABLE II.

TABLE II

PARAMETERS SETTING OF CLASSIFIERS

\begin{tabular}{lll}
\hline Classifier & Kernel function & parameters \\
\hline SVM & RBF kernel & $(C, \gamma)=(1,0.0216)$ \\
\hline NN & Identity function & $(I, H, O)=(2,7,10)$ \\
\hline
\end{tabular}

Fig.6 shows the comparison of the RF algorithm with SVM and NN. The experimental results show that the recognition rates of SVM and NN are $90.89 \%$ and $89.1 \%$, respectively, which are both lower than the recognition rate of RF. It can be seen clearly that the recognition rates of motion 1 are relatively close. Besides, SVM has the highest recognition rate for motions 7 and 8 , indicating the good performance. The standard deviation reflects the fluctuation of the recognition rate of the same motion by different algorithms. Overall, the recognition results indicate that the hand motion recognition system based on this method is more effective than other two algorithms.

\section{Conclusions}

This paper presented a intention identification of complex hand motion based SEMG signal. It exhibited the detailed presentation of the hand motion recognition system, including hand motions design, signal processing, trained RF classification modeling. In the part of signal processing, the reasonable SEMG features as training samples were obtained 


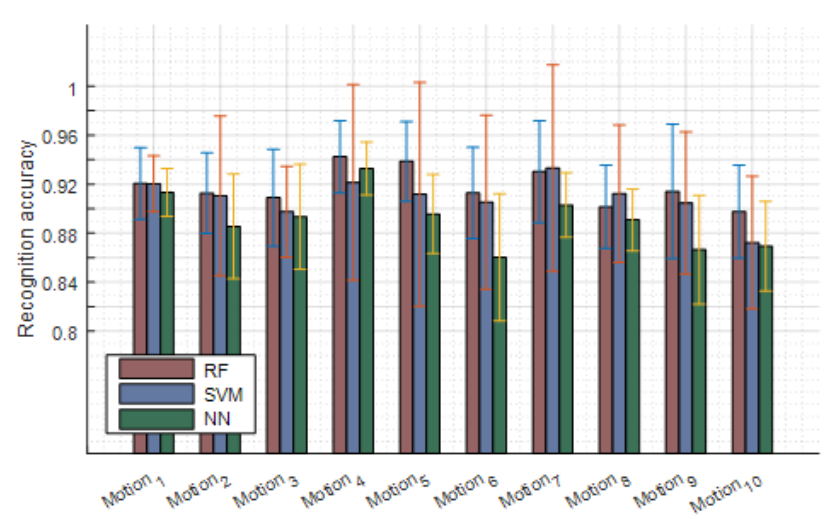

Fig. 6: The comparison of three algorithms

based on signal denoising and feature extraction. The RF classifier was used to classify the different hand motions. The experimental results shows that the proposed hand motion recognition system has a better performance for complex motion classification. The total recognition rate is $91.67 \%$. In the future, more data analysis and results discussion such as the comparison of different classifiers, different features, etc. will be studied.

\section{ACKNOWLEDGMENT}

This work is partially supported by Natural Science Foundation of China (Grant No.). Furthermore, the authors would like to acknowledge the reviewers for their valuable comments and suggestions that helped to improve the quality of the manuscript.

\section{REFERENCES}

[1] Mishra V K, Bajaj V, Kumar A, et al. An efficient method for analysis of EMG signals using improved empirical mode decomposition[J]. AEUInternational Journal of Electronics and Communications, 2017, 72: 200209

[2] Poh M Z, Swenson N C, Picard R W. A wearable sensor for unobtrusive, long-term assessment of electrodermal activity[J]. IEEE transactions on Biomedical engineering, 2010, 57(5): 1243-1252.

[3] Cerone G L, Botter A, Gazzoni M. A Modular, Smart, and Wearable System for High Density sEMG Detection[J]. IEEE Transactions on Biomedical Engineering, 2019.

[4] Xue Y, Ju Z, Xiang K, et al. Multiple sensors based hand motion recognition using adaptive directed acyclic graph[J]. Applied Sciences, 2017, 7(4): 358.

[5] Xue Y, Ju Z, Xiang K, et al. Multimodal human hand motion sensing and analysis-a review[J]. IEEE Transactions on Cognitive and Developmental Systems, 2018.

[6] Ju Z, Ouyang G, Wilamowska-Korsak M, et al. Surface EMG based hand manipulation identification via nonlinear feature extraction and classification[J]. IEEE Sensors Journal, 2013, 13(9): 3302-3311.

[7] Lu Y, Ju Z, Liu Y, et al. Time series modeling of surface EMG based hand manipulation identification via expectation maximization algorithm[J]. Neurocomputing, 2015, 168: 661-668.

[8] Yin X, Chen Q. Deep metric learning autoencoder for nonlinear temporal alignment of human motion[C]//2016 IEEE International Conference on Robotics and Automation (ICRA). IEEE, 2016: 2160-2166.

[9] Ding Z, Yang C, Tian Z, et al. sEMG-Based Gesture Recognition with Convolution Neural Networks[J]. Sustainability, 2018, 10(6): 1865.

[10] Grond F, Diebner H H, Sahle S, et al. A robust, locally interpretable algorithm for Lyapunov exponents[J]. Chaos, Solitons \& Fractals, 2003, 16(5): 841-852.
[11] Rosenstein M T, Collins J J, De Luca C J. A practical method for calculating largest Lyapunov exponents from small data sets[J]. Physica D: Nonlinear Phenomena, 1993, 65(1-2): 117-134.

[12] Kantz H. A robust method to estimate the maximal Lyapunov exponent of a time series[J]. Physics letters A, 1994, 185(1): 77-87.

[13] Fujisaka H. Statistical dynamics generated by fluctuations of local Lyapunov exponents[J]. Progress of theoretical physics, 1983, 70(5): 1264-1275.

[14] Key E. Computable examples of the maximal Lyapunov exponent[J]. Probability theory and related fields, 1987, 75(1): 97-107.

[15] Guo Y, Naik G R, Huang S, et al. Nonlinear multiscale Maximal Lyapunov Exponent for accurate myoelectric signal classification[J]. Applied Soft Computing, 2015, 36: 633-640.

[16] N.E. Huang, M.L. Wu, W. Qu, S.R. Long, S.S. Shen, Applications of HilbertHuangtransform to non-stationary financial time series analysis, Appl. Stoch. ModelsBus. Ind. 19 (2003) 245268.

[17] A. Fraser and H. Swinney, Independent coordinates for strange attractors from mutual information, Phys. Rev. A, vol. 33, no. 2, pp. 11341140, Feb. 1986.

[18] L. Cao, Practical method for determining the minimum embedding dimension of a scalar time series, Phys. D, Nonlinear Phenomena, vol. 110, nos. 12, pp. 4350, Dec. 1997.

[19] Xue Y, Ju Z, Xiang K, et al. Dexterous hand motion classification and recognition based on multimodal sensing[C]//International Conference on Intelligent Robotics and Applications. Springer, Cham, 2017: 450-461.

[20] Han S, Pool J, Tran J, et al. Learning both weights and connections for efficient neural network[C]//Advances in neural information processing systems. 2015: 1135-1143. 